COMMUNICATIONS IN

ANALYSIS AND GEOMETRY

Volume 15, Number 1, 147-174, 2007

\title{
An invariant for triples in the Shilov boundary of a bounded symmetric domain
}

\author{
JEAN-LOUIS CLERC
}

Let $\mathcal{D}$ be a bounded symmetric domain, $G$ its group of biholomorphic diffeomorphisms and $S$ its Shilov boundary. We define a function $\iota: S \times S \times S \longrightarrow \mathbb{R}$, which is invariant under $G$. This invariant generalizes the Maslov index as defined for the Shilov boundary of a tube-type domain (see $[2,3,4]$ ) and the angular invariant constructed by E. Cartan for the unit sphere in $\mathbb{C}^{2}$ (see [1]).

\section{Introduction}

In a paper published in 1932 (cf. [1]), Elie Cartan studied the geometry of the unit sphere $S$ in $\mathbb{C}^{2}$,

$$
S=\left\{(x, y) \in \mathbb{C}^{2} \mid x \bar{x}+y \bar{y}=1\right\}
$$

under the action of the group $G$ of holomorphic transformations (defined in a neighborhood of $S$ ) preserving $S$. A better understanding of the geometry of $S$ is achieved by using another realization of $S$. On $\mathbb{C}^{3}$ consider the Hermitian form $h$ given by

$$
h\left((z, x, y),\left(z^{\prime}, x^{\prime}, y^{\prime}\right)\right)=z \overline{z^{\prime}}-x \overline{x^{\prime}}-y \overline{y^{\prime}} .
$$

The space $\mathcal{S}$ of complex lines in $\mathbb{C}^{3}$, which are istropic with respect to $h$, is in one-to-one correspondence with $S$ by the mapping

$$
S \ni(x, y) \longmapsto \mathbb{C}(1, x, y) \in \mathcal{S}
$$

The group $S U(h) \simeq S U(1,2)$ acts on $\mathbb{C}^{3}$ preserving the form $h$ and hence the isotropic lines. This gives raise to an action of $G=P S U(1,2)$ on $\mathcal{S}$, which, after conjugation by the correspondence between $\mathcal{S}$ and $S$, gives the classical homographic action of $G$ on $S$. 
Among several other results, Cartan constructed an invariant for triples in $S$. For $v_{1}, v_{2}, v_{3} \in \mathbb{C}^{3} \backslash\{0\}$, consider the complex number

$$
J\left(v_{1}, v_{2}, v_{3}\right)=h\left(v_{1}, v_{2}\right) h\left(v_{2}, v_{3}\right) h\left(v_{3}, v_{1}\right) .
$$

Observe that, under replacement of $v_{1}$ by $\lambda_{1} v_{1}$ (resp. $v_{2}$ by $\lambda_{2} v_{2}, v_{3}$ by $\lambda_{3} v_{3}$ ), where $\lambda_{1}$ (resp. $\lambda_{2}, \lambda_{3}$ ) is any nonzero complex number, the quantity $J$ is multiplied by $\left|\lambda_{1}\right|^{2}\left|\lambda_{2}\right|^{2}\left|\lambda_{3}\right|^{2}$, hence the argument of $J$ depends only on the triple of complex lines $\left(\mathbb{C} v_{1}, \mathbb{C} v_{2}, \mathbb{C} v_{3}\right)$. To be more precise (and this observation will be of importance later on), observe that, if $v_{1}$ and $v_{2}$ are two isotropic nonzero vectors in $\mathbb{C}^{3}$, then $h\left(v_{1}, v_{2}\right)=0$ implies that $\mathbb{C} v_{1}+\mathbb{C} v_{2}$ is totally isotropic for $h$, and hence $v_{1}$ and $v_{2}$ are proportional. So, if $\sigma_{1}, \sigma_{2}, \sigma_{3}$ are three distinct points in $\mathcal{S}$, define, following Cartan

$$
j\left(\sigma_{1}, \sigma_{2}, \sigma_{3}\right)=\arg J\left(v_{1}, v_{2}, v_{3}\right)
$$

where $v_{1}$ (resp. $v_{2}, v_{3}$ ) is any nonzero vector of $\sigma_{1}$ (resp. $\sigma_{2}, \sigma_{3}$ ). As the quantity $J$ is invariant under $S U(h)$, the quantity $j$ is invariant under the action of $G$. As $h$ is Hermitian symmetric, one more property of $j$ is that it is skew symmetric with respect to permutation of the points $\sigma_{1}, \sigma_{2}, \sigma_{3}$. Notice for further reference that this suggests to extend the definition of $j$ by requiring that the value of $j\left(\sigma_{1}, \sigma_{2}, \sigma_{3}\right)$ is 0 if (at least) two points among $\sigma_{1}, \sigma_{2}, \sigma_{3}$ coïncide.

Obviously, Cartan's construction is valid as well for the unit sphere in $\mathbb{C}^{n}$, under the action of $G=P S U(1, n)$. The case $n=1$ is even of special interest. As before, the unit sphere $S=\{z \in \mathbb{C},|z|=1\}$ is in one-to-one correspondence with the space of isotropic lines in $\mathbb{C}^{2}$ for the Hermitian form $h$ on $\mathbb{C}^{2}$ given by

$$
h\left((z, x),\left(z^{\prime}, x^{\prime}\right)\right)=z \overline{z^{\prime}}-x \overline{x^{\prime}}
$$

the correspondence being

$$
e^{i \theta} \longmapsto \mathbb{C}\left(1, e^{i \theta}\right)
$$

The remarkable fact (not immediately trivial) is that the quantity

$$
J\left(v_{1}, v_{2}, v_{3}\right)=h\left(v_{1}, v_{2}\right) h\left(v_{2}, v_{3}\right) h\left(v_{3}, v_{1}\right),
$$

or the quantity

$$
j\left(e^{i \theta_{1}}, e^{i \theta_{2}}, e^{i \theta_{3}}\right)=\left(1-e^{i\left(\theta_{1}-\theta_{2}\right)}\right)\left(1-e^{i\left(\theta_{2}-\theta_{3}\right)}\right)\left(1-e^{i\left(\theta_{3}-\theta_{1}\right)}\right)
$$


is pure imaginary for any isotropic vectors $v_{1}, v_{2}, v_{3}$ (resp. for any $\theta_{1}, \theta_{2}, \theta_{3}$ $\in \mathbb{R}$ ). Hence, in this case, Cartan's invariant takes (on triples of distinct points in $S$ ) only two values: $-\frac{\pi}{2}$ and $\frac{\pi}{2}$. They correspond to the two orbits under $G$ in the space of (proper) triples, corresponding to the orientation of the triple which may or may not agree with the counterclockwise orientation of the unit circle. Another model for the unit circle is the Lagrangian manifold of real lines in the symplectic space $\mathbb{R}^{2}$ under the action of the group $G=S p(\mathbb{R}) \simeq S L(2, \mathbb{R})$. In this interpretation, Cartan's invariant (up to a factor $-\frac{\pi}{2}$ ) is nothing but the Maslov triple index (see [2] for details).

In this paper, we consider any bounded symmetric domain $\mathcal{D}$. Let $G$ be (the neutral component of) its group of biholomorphic diffeomorphisms, and let $S$ be its Shilov boundary. The action of any element $g \in G$ extends to a neighborhood of $\overline{\mathcal{D}}$ and preserves $S$. For triples $\left(\sigma_{1}, \sigma_{2}, \sigma_{3}\right) \in S^{3}$, we construct an invariant for the action of $G$, which generalizes Cartan's invariant. If $\mathcal{D}$ happens to be of tube type, this program was achieved in [3], following previous work in collaboration with Orsted (see $[2,4]$ ). As it was the case for the unit circle in $\mathbb{C}$ (which is the Shilov boundary of the tube-type domain $\mathcal{D}=\{z \in \mathbb{C},|z|<1\}$ ), the invariant in the tube-type case is always an integer (after normalization), and this corresponds to the geometric fact that $G$ has a finite number of (open) orbits in $S^{3}$. This is not the case when $\mathcal{D}$ is not of tube type.

A further study of Cartan's invariant can be found in [5]. For the classical theory of the Maslov index, see [6]. For the case of the Stiefel manifold, viewed as the Shilov boundary of the unit ball in $\operatorname{End}\left(\mathbb{C}^{p}, \mathbb{C}^{q}\right)$, a more general invariant was studied in [7]. For the same space, the paper [8] gives a direct generalization of Cartan's invariant and some interesting geometric applications.

Let us sketch the construction of the invariant. Let first $z_{1}, z_{2}, z_{3}$ be three points in $\mathcal{D}$. Form the oriented geodesic triangle $T\left(z_{1}, z_{2}, z_{3}\right)$, and consider any surface $\Sigma$ in $\mathcal{D}$ which has this triangle as boundary. We may integrate the Kähler form $\omega$ of the domain $\mathcal{D}$ on $\Sigma$ and obtain a real number

$$
\varphi\left(z_{1}, z_{2}, z_{3}\right)=\int_{\Sigma} \omega
$$

(not depending on $\Sigma$ as the Kähler form is closed), which we call the symplectic area of the triangle $T\left(z_{1}, z_{2}, z_{3}\right)$. As the Kähler form is invariant under $G$, this gives an invariant for triples in $\mathcal{D}$. Now for $\sigma_{1}, \sigma_{2}, \sigma_{3} \in S^{3}$, define

$$
\iota\left(\sigma_{1}, \sigma_{2}, \sigma_{3}\right)=\frac{1}{\pi} \lim _{z_{j} \longrightarrow \sigma_{j}} \varphi\left(z_{1}, z_{2}, z_{3}\right) .
$$


The difficulty is to show that there is actually a limit. In the generic case, i.e., for mutually transverse triples (see precise definition in [4]), the limit exists without any restriction. However, for the singular triples, one needs to restrict the way $z_{j}$ approaches $\sigma_{j}$. This is where the notion of $\Gamma$-radial convergence is required. Note that this restricted approach to the boundary is already needed in the case of the unit circle, for triples in which two points coincide. In fact, let $\sigma_{1}$ and $\sigma_{2}=\sigma_{3}$ be points of the unit circle. As $z_{1}$ approaches $\sigma_{1}, z_{2}$ and $z_{3}$ approach $\sigma_{2}$, the invariant $\varphi\left(z_{1}, z_{2}, z_{3}\right)$ (which is nothing but the oriented area of the geodesic triangle $T\left(z_{1}, z_{2}, z_{3}\right)$ for the Poincaré metrics of the disc) may approach any value between $-\pi$ and $\pi$. However if one demands that $z_{2}$ and $z_{3}$ approach $\sigma_{2}=\sigma_{3}$ along curves which are radial (= normal to $S$ ) at $\sigma_{2}$, then $\varphi\left(z_{1}, z_{2}, z_{3}\right.$ ) tends to 0 , which, as noticed earlier, ought to be the value of $\iota\left(\sigma_{1}, \sigma_{2}, \sigma_{3}\right)$. In higher rank, the corresponding statement requires more work.

As a bonus to this definition/theorem, we obtain a further property of the invariant (for original Cartan's invariant, this property had been noticed in $[5$, ch.7]). It satisfies a cocycle property, namely

$$
\iota\left(\sigma_{1}, \sigma_{2}, \sigma_{3}\right)=\iota\left(\sigma_{1}, \sigma_{2}, \sigma_{4}\right)+\iota\left(\sigma_{2}, \sigma_{3}, \sigma_{4}\right)+\iota\left(\sigma_{3}, \sigma_{1}, \sigma_{4}\right)
$$

for any $\sigma_{1}, \sigma_{2}, \sigma_{3}, \sigma_{4} \in S$. In fact, the same property is already satisfied by the function $\varphi\left(z_{1}, z_{2}, z_{3}\right)$, and it is merely a consequence of Stokes formula and the fact that the Kähler form is closed.

Section 2 introduces notation and results of the geometry of bounded symmetric domains, both in the bounded and the unbounded realizations. The presentation uses the theory of positive Hermitian Jordan triple systems. Section 3 introduces the wedge structure on $S$ and the related notion of $\Gamma$-radial convergence at a point of the Shilov boundary. Section 4 contains a technical result, written in the framework of Euclidean Jordan algebras and their complexifications, which might be of independent interest. Section 5 gives the construction and states the main properties of the invariant $\iota$. Section 6 gives a geometric description of the triples in $S$ corresponding to the maximal value of the invariant $\iota$.

\section{Bounded symmetric domains and positive Hermitian Jordan triple systems}

This section is devoted to a detailed presentation of bounded symmetric domains, using the theory of positive Hermitian Jordan triple systems. For general references, see [9]. The link of this point of view with the more 
classical Lie group theory approach (see [10]) is exposed in [11] or in [12]. The presentation in [13] is also relevant. For the theory of Euclidean Jordan algebras, see [14].

A Jordan triple system $W$ is a real vector space together with a trilinear map $\{., .,\}:. W \times W \times W \longrightarrow W$, which satisfies

$$
\{a, b,\{x, y, z\}\}=\{\{a, b, x\}, y, z\}-\{x,\{b, a, y\}, z\}+\{x, y,\{a, b, z\}\}
$$

for all $a, b, x, y, z \in W$.

For $x, y \in W$ denote by $x \square y$ the linear endomorphism of $W$ defined by

$$
(x \square y) z=\{x, y, z\}
$$

and by $Q(x)$ the linear endomorphism of $W$ defined by

$$
Q(x) z=\{x, z, x\}
$$

Define the trace form $\tau$ on $W$ by

$$
\tau(x, y)=\operatorname{tr}(x \square y)
$$

The Jordan triple system is said to be nondegenerate if, as a bilinear form, $\tau$ is nondegenerate. If this is the case, then $\tau$ is symmetric. Moreover, for $x, y \in W$

$$
(x \square y)^{t}=y \square x,
$$

where, for $A \in \operatorname{End}(W), A^{t}$ is used for the transpose of $A$ with respect to $\tau$.

A nondegenerate Hermitian Jordan triple system $\mathbb{W}$ is a complex vector space, together with a map $\{., .,\}:. \mathbb{W} \times \mathbb{W} \times \mathbb{W} \longrightarrow \mathbb{W}$, such that $\{x, y, z\}$ is complex linear in $x$ and $z$, conjugate-linear in $y$, and such that $\mathbb{W} \mathbb{R},\{., .,$.$\} is a nondegenerate Jordan triple system, where \mathbb{W}^{\mathbb{R}}$ stands for $\mathbb{W}$ viewed as a real vector space. Abusing somewhat notation, set

$$
\tau(x, y)=\operatorname{tr}_{\mathbb{C}}(x \square y),
$$

which is then a nondegenerate Hermitian form on $\mathbb{W}$. If if $\tau$ happens to be positive definite, then $\mathbb{W}$ is said to be a positive Hermitian Jordan triple 
system (PHJTS). Now, for $x, y \in \mathbb{W}$,

$$
(x \square y)^{*}=y \square x,
$$

where $A^{*}$ stands for the complex adjoint of $A$ with respect to the Hermitian form $\tau$.

Let $\mathbb{W}$ be a PHJTS. If $x$ is an element of $\mathbb{W}$, then its odd powers $x^{(2 n+1)}$ are defined by the induction formula

$$
x^{(2 n+1)}=\left\{x^{2 n-1}, x, x\right\} .
$$

An element $c \in \mathbb{W}$ is said to be a tripotent if $c^{(3)}=\{c, c, c\}=c$.

There is a (partial) order relation on tripotents. For $c, d$, two tripotents of $\mathbb{W}$, the relation $c \prec d$ is true if and only if there exists a tripotent $c^{\prime}$, such that

(i) $c \square c^{\prime}=0$ (orthogonality of $c$ and $c^{\prime}$ )

(ii) $d=c+c^{\prime}$.

A nonzero tripotent is said to be primitive if it is minimal for this order among nonzero tripotents. Any tripotent $c$ can be written as a sum of pairwise orthogonal primitive tripotents, say $c=c_{1}+c_{2}+\cdots+c_{k}$. The number $k$ of primitive tripotents in such a decomposition of $c$ depends only on $c$ and is called the rank of $c$.

One of the main results in the theory of PHJTS is the spectral theorem.

Proposition 2.1. Every $x \in \mathbb{W}$ can be written uniquely

$$
x=\sum_{j=1}^{k} \lambda_{j} c_{j},
$$

where $\left(c_{j}\right)_{1 \leq j \leq k}$ are paiwise orthogonal nonzero tripotents which are real linear combinations of powers of $x$, and $\lambda_{j}$ satisfy

$$
0<\lambda_{1}<\lambda_{2}<\cdots<\lambda_{k} .
$$

The identity (2.3) is called the spectral decomposition of $x$. The $\lambda_{j}$ are called the eigenvalues of $x$. The largest eigenvalue is the spectral norm of $x$, denoted by $|x|$. As notation suggests, the map $x \mapsto|x|$ can be shown to be a (complex Banach) norm on $\mathbb{W}$. Moreover, $|x|=\|Q(x)\|=\frac{1}{2}\|x \square x\|$, where the operator norm $\|A\|$ for any $A \in \operatorname{End}_{\mathbb{R}}(\mathbb{W})$ is computed with respect to the inner product on $\mathbb{W}$ induced by $\tau$. 
Theorem 2.2. The unit ball of $(\mathbb{W},|\cdot|)$ is a bounded symmmetric domain. Conversely, any bounded symmetric domain is biholomorphically equivalent to such a unit ball.

In other words, any bounded symmetric domain can be realized as the unit ball for the spectral norm of some PHJTS. For a proof of this important result, see [9].

Let $G=G(\mathcal{D})$ be the neutral component of the group of holomorphic diffeomorphisms of $\mathcal{D}$. It is a semi-simple Lie group, which acts transitively on $\mathcal{D}$. Let $K$ be the stabilizer of 0 in $G$. The subgroup $K$ is a maximal subgroup of $G$, and $\mathcal{D} \simeq G / K$. Denote by $r$ be the rank of $\mathcal{D}$ as a symmetric space.

Let $c$ be a nonzero tripotent of $\mathbb{W}$. Then $\mathbb{W}$ decomposes as

$$
\mathbb{W}=\mathbb{W}_{2} \oplus \mathbb{W}_{1} \oplus \mathbb{W}_{0}
$$

where $\mathbb{W}_{j}=\mathbb{W}_{j}(c)$ is the eigenspace of $c \square c$ corresponding to the eigenvalue $\frac{1}{2} j$. The $\mathbb{W}_{j}$ are pairwise orthogonal and satisfy the rule

$$
\left\{\mathbb{W}_{j}, \mathbb{W}_{k}, \mathbb{W}_{l}\right\} \subset \mathbb{W}_{j-k+l}, \quad\left\{\mathbb{W}_{2}, \mathbb{W}_{0}, \mathbb{W}\right\}=\left\{\mathbb{W}_{0}, \mathbb{W}_{2}, \mathbb{W}\right\}=\{0\}
$$

This decomposition of $\mathbb{W}$ will be referred to as the Peirce decomposition with respect to $c$.

Proposition 2.3. Let $e$ be a tripotent in $\mathbb{W}$. The following are equivalent

(i) e is a maximal tripotent

(ii) e has rank $r$

(iii) $\mathbb{W}_{0}(e)=\{0\}$

(iv) e is an extremal point of the convex set $\overline{\mathcal{D}}$.

The set of all maximal tripotents is a compact submanifold $S$ of $\mathbb{W}$. It is the Shilov boundary of $\mathcal{D}$ in the sense of complex analysis. It is the unique closed orbit of $G$ in $\partial \mathcal{D}$, and the group $K$ is already transitive on $S$.

A maximal family of orthogonal primitive tripotents in $\mathbb{W}$ is called a Jordan frame. It consists of $r$ (primitive, mutually orthogonal) tripotents $c_{1}, c_{2}, \ldots, c_{r}$. 
For $x, y \in \mathbb{W}$, set

$$
B(x, y)=\operatorname{Id}-x \square y+Q(x) Q(y) .
$$

Observe that $B(x, y)$ is a $\mathbb{C}$-linear endomorphism of $\mathbb{W}$. It is a holomorphic polynomial with respect to the variable $x$ and antiholomorphic with respect to $y$. If $B(x, y)$ is invertible, then $x$ and $y$ are said to be transverse and this relation is denoted by $x \top y$. It is a symmetric relation.

There is an alternative realization of the same spaces, called the unbounded realization, which is obtained through a Cayley transform.

Fix $e$ a maximal tripotent in $\mathbb{W}$. Then $\mathbb{W}_{2}=\mathbb{W}_{2}(e)$ has a natural structure of complex Jordan algebra for the Jordan product defined by

$$
x \circ y=\{x, e, y\} .
$$

The element $e$ is the neutral element of this Jordan algebra. Moreover, $Q(e)$ maps $\mathbb{W}_{2}$ into itself, and can be shown to be a conjugate-linear involution of $\mathbb{W}_{2}$. For $a \in \mathbb{W}_{2}$, set $a^{*}=Q(e) a$. The space of fixed points of this involution $U=\left\{a \in \mathbb{W}_{2} \mid a=a^{*}\right\}$ inherits a structure of real Euclidean Jordan algebra. Standard notation for Jordan algebra (see [14]) is used freely throughout the paper, such as $\Omega \subset U$ for the (open) cone of squares, $L(x)$ for the multiplication by $x, P(x)=2 L(x)^{2}-L\left(x^{2}\right)$ for the quadratic operator associated to $x$ and det for the determinant polynomial on $U$ (or $\mathbb{W}_{2}$ ).

To any $a \in \mathbb{W}_{2}$ associate the endomorphism $\Phi(a)$ of $\mathbb{W}_{1}$ given by

$$
\Phi(a) v=2\{a, e, v\}
$$

Then for $a \in U$, the endomorphism $\Phi(a)$ is self-adjoint with respect to the inner product on $\mathbb{W}_{1}$ induced by the form $\tau$, and satisfies

$$
\Phi(a \circ b)=\frac{1}{2}(\Phi(a) \Phi(b)+\Phi(b) \Phi(a)), \quad \Phi(e)=\operatorname{Id}_{\mathbb{W}_{1}} .
$$

In other words, $\Phi$ is a representation of the Euclidean Jordan algebra $U$ on $\mathbb{W}_{1}$. Finally, let $\Psi: \mathbb{W}_{1} \times \mathbb{W}_{1} \longrightarrow \mathbb{W}_{2}$ be the bilinear map defined by

$$
\Psi\left(v, v^{\prime}\right)=\left\{v, v^{\prime}, e\right\}
$$

Then $\Psi$ is Hermitian and positive definite in the sense that

$$
\Psi\left(v, v^{\prime}\right)=\Psi\left(v^{\prime}, v\right)^{*}, \quad \Psi(v, v) \in \bar{\Omega}, \quad \Psi(v, v)=0 \Longleftrightarrow v=0 .
$$


A further property of $\Phi$ and $\Psi$ is the following relation

$$
\Psi\left(\Phi(x) v, \Phi(x) v^{\prime}\right)=P(x)\left(\Psi\left(v, v^{\prime}\right)\right)
$$

for all $x \in U, v, v^{\prime} \in \mathbb{W}_{1}$.

Proposition 2.4. Let e be a maximal tripotent. Let $\mathbb{W}=\mathbb{W}_{2} \oplus \mathbb{W}_{1}$ be the corresponding Peirce decomposition of $\mathbb{W}$. Then $x=x_{2}+x_{1}$ is transverse to $e$ if and only if $\operatorname{det}\left(e-x_{2}\right) \neq 0$.

Proof. A routine computation shows that $B(x, e)$ as an endomorphism of $\mathbb{W}$ has the following block realization with respect to the decomposition $\mathbb{W}=\mathbb{W}_{2} \oplus \mathbb{W}_{1}$ :

$$
B(x, e)=\left(\begin{array}{cc}
\operatorname{Id}_{2}-2 L\left(x_{2}\right)+P\left(x_{2}\right) & 0 \\
* & \operatorname{Id}_{1}-\Phi\left(x_{2}\right)
\end{array}\right) .
$$

As $\operatorname{Id}_{2}-2 L\left(x_{2}\right)+P\left(x_{2}\right)=P\left(e-x_{2}\right)$ and $\operatorname{Id}_{1}-\Phi\left(x_{2}\right)=\Phi\left(e-x_{2}\right)$ and $\Phi(z)$ is invertible if and only if $z$ is invertible (and then $\Phi(z)^{-1}=\Phi\left(z^{-1}\right)$ ), it is clear that the invertibility in $\mathbb{W}_{2}$ of $e-x_{2}$ is the necessary and sufficient condition for the invertibility of $B(x, e)$. Proposition 2.4 follows.

Corresponding to the data $U, \Omega, \Psi, \mathbb{W}_{1}$, let ${ }^{\gamma} \mathcal{D}$ be the Siegel domain of the second kind defined by

$$
{ }^{\gamma} \mathcal{D}=\left\{(x+i y, v), x, y \in U, v \in \mathbb{W}_{1} \mid y-\Psi(v, v) \in \Omega\right\}
$$

The (unbounded) domain ${ }^{\gamma} \mathcal{D}$ is biholomorphically equivalent to the domain $\mathcal{D}$. The correspondence between the two domains has an explicit description as a Cayley transform $\gamma=\gamma_{e}$, which is defined (as a rational map on $\mathbb{W}_{2} \times$ $\left.\mathbb{W}_{1}\right)$ by

$$
\gamma_{e}\left(x_{2}, x_{1}\right)=\left(i\left(e+x_{2}\right)\left(e-x_{2}\right)^{-1}, \Phi\left(\left(e-x_{2}\right)^{-1}\right) x_{1}\right)
$$

For $x=x_{2}+x_{1} \in \mathcal{D}, e-x_{2}$ is invertible in $\mathbb{W}_{2}$, and hence the Cayley transforms is well-defined on $\mathcal{D}$. More generally, the Cayley transform is defined precisely on the elements $x \in \mathbb{W}$ which are transverse to $e$ (thanks to Proposition 2.4). In particular, the Cayley transform is defined on

$$
S_{e}^{\top}=\{x \in S \mid x \top e\}=\left\{x=x_{2}+x_{1} \in S \mid \operatorname{det}\left(e-x_{2}\right) \neq 0\right\} .
$$


This is a dense open set in $S$, and its image under $\gamma_{e}$ is given by

$$
{ }^{\gamma} S^{\prime}=\left\{(x+i \Psi(v, v), v) \mid x \in U, v \in \mathbb{W}_{1}\right\} .
$$

\section{The wedge structure on $S$ and the notion of $\Gamma$-radial convergence}

Let $M$ be a manifold. For each point $x \in M$, let $\Gamma_{x}$ be a nontrivial convex open cone in the tangent space $T_{x} M$ at $x$, and assume that the cone $\Gamma_{x}$ depends smoothly on $x$. Then we say that $M$ is given a wedge structure. The concept of causal structure is more common. This is the case where one demands that the cone be proper (its closure does not contain any line), but it is important for our purpose not to make this requirement. In the case at hand, we might also call this structure a weakly causal structure.

A wedge diffeomorphism is a diffeomorphism $F: M \longrightarrow M$ such that, at each point $x \in M$, the differential $D F(x)$ maps the cone $\Gamma_{x} \subset T_{x} M$ into the cone $\Gamma_{F(x)} \subset T_{F(x)} M$. If $G$ is a Lie group acting by diffeomorphisms on $M$, the wedge structure is said to be invariant under $G$ if each $g \in G \subset$ $\operatorname{Diff}(M)$ is a wedge diffeomorphism. Assume moreover that the action of $G$ on $M$ is transitive. Choose a base point $o$ in $M$, and let $H=G_{o}$ be the stabilizer of $o$ in $G$. An invariant wedge structure on $M$ is completely determined by the cone $\Gamma_{o} \subset T_{o} M \simeq \mathfrak{g} / \mathfrak{h}$. This cone has to be invariant under the tangent action of $\operatorname{Ad}(H)$ on $\mathfrak{g} / \mathfrak{h}$. But conversely, given such an invariant cone $\Gamma_{o} \subset \mathfrak{g} / \mathfrak{h}$, one can unambiguously propagate that cone to endow $M$ with a $G$-invariant wedge structure.

The Shilov boundary $S$ of a bounded symmetric space has a natural wedge structure. On the Shilov boundary of a tube-type domain, it has been known that there is a natural causal structure (see $[15,16])$. From another point of view, for any bounded symmetric domain, there is a natural Cauchy-Riemann structure on $S$. The wedge structure we will consider on $S$ is a mixed version of these two structures.

The wedge structure will be described first in the unbounded realization of $\mathcal{D}$. For simplicity, let us modify the notation by setting $\mathbb{U}=U \otimes_{\mathbb{R}} \mathbb{C}=\mathbb{W}_{2}$ and $\mathbb{V}=\mathbb{W}_{1}$. With this notation, recall (2.12)

$$
{ }^{\gamma} \mathcal{D}=\{(x+i y, v) \mid x, y \in U, v \in \mathbb{V}, y-\Psi(v, v) \in \Omega\}
$$

whereas

$$
{ }^{\gamma} S^{\prime}=\{(x+i y, v) \mid x, y \in U, v \in \mathbb{V}, y=\Psi(v, v)\}
$$


Choose $o=(0,0)$ as origin in ${ }^{\gamma} S^{\prime}$. The tangent space to ${ }^{\gamma} S$ at $o$ is a real vector subspace of $\mathbb{W}$, given by the condition $y=0$, hence realized as $U \oplus \mathbb{V}$. Then let $\Gamma_{o}=\Omega+\mathbb{V}$.

Proposition 3.1. Let $g$ be any holomorphic diffeomorphism of ${ }^{\gamma} \mathcal{D}$, such that $g(o)=o$. Then $D g(o) \Gamma_{o} \subset \Gamma_{o}$.

Recall first that any holomorphic diffeomorphism of $\mathcal{D}$ extends to a neighborhood of $\overline{\mathcal{D}}$, and similarly for ${ }^{\gamma} \mathcal{D}$. Let $\omega \in \Omega$, and consider the path

$$
\gamma:[0,1] \longrightarrow i U, \quad \gamma(t)=i t \omega
$$

For $t>0, \gamma(t) \in \mathcal{D}$, so that

$$
g(\gamma(t))=\gamma_{1}(t)=\left(x_{1}(t)+i y_{1}(t), v_{1}(t)\right) \in^{\gamma} \mathcal{D}
$$

So, for any $t>0, y_{1}(t)-\Psi\left(v_{1}(t), v_{1}(t)\right) \in \Omega$, and a fortiori $y_{1}(t) \in \Omega$. Hence, $\dot{y}_{1}(0) \in \bar{\Omega}$ and

$$
\dot{\gamma}_{1}(0)=D g(o) i \omega \in U \oplus i \bar{\Omega} \oplus \mathbb{V} .
$$

But as $D g(o)$ is $\mathbb{C}$-linear and maps the tangent space to $S$ at $o$ into itself, we also have

$$
D g(o)(U \oplus \mathbb{V}) \subset U \oplus \mathbb{V}, \quad D g(o)(\mathbb{V}) \subset \mathbb{V} .
$$

Hence, after multiplication by $i$,

$$
D g(o)(i \Omega) \subset i U \oplus \mathbb{V},
$$

which together with (3.1) implies

$$
D g(o)(i \Omega) \subset i \bar{\Omega} \oplus \mathbb{V} .
$$

But $D g(o)$ is invertible, and hence the image of an open set is open, so that

$$
D g(o)(i \Omega) \subset i \Omega \oplus \mathbb{V}
$$

which, after multiplication by $i$ (recalling that $g$ is holomorphic), implies

$$
D g(o)(\Omega \oplus \mathbb{V}) \subset \Omega \oplus \mathbb{V} .
$$


Thus, the wedge $\Omega \oplus \mathbb{V}$ is invariant by the stabilizer of $o$ in ${ }^{\gamma} G=\gamma \circ G \circ \gamma^{-1}$, and hence defines an invariant wedge structure on ${ }^{\gamma} S^{\prime}$. The corresponding description of the wedge structure on $S$ is obtained by inverse Cayley transform.

Proposition 3.2. There exists a unique $G$-invariant wedge structure $\left(\Gamma_{\sigma}\right.$, $\sigma \in S)$ on $S$, such that $\Gamma_{-e}=-i \Omega \oplus \mathbb{V}$.

Sketch of the proof. As a consequence of (2.13), one has $\gamma_{e}(-e)=o$ and one can show that

$$
D \gamma(-e)=\left(\frac{i}{2} \operatorname{Id}_{\mathbb{U}}, \frac{1}{4} \operatorname{Id}_{\mathbb{V}}\right)
$$

The proposition follows, by taking the inverse image of the cone $\Omega \oplus \mathbb{V}$.

A curve $(\gamma(t), 0 \leq t \leq 1)$ is said to be $\Gamma$-radial at some point $\sigma \in S$ if

$$
\gamma(0)=\sigma, \quad \gamma(t) \in \mathcal{D} \text { for } 0<t \leq 1, \text { and } \dot{\gamma}(0) \in i \Gamma_{\sigma} .
$$

Proposition 3.3. Let $\sigma \in S$, and let $(\gamma(t), 0 \leq t \leq 1)$ be a $\Gamma$-radial curve at $\sigma$. Let $g \in G$. Then $(g(\gamma(t)), 0 \leq t \leq 1)$ is a $\Gamma$-radial curve at $g(\sigma)$.

As the differential of $g$ at $\sigma$ is $\mathbb{C}$-linear, Proposition 3.3 is a consequence of the invariance of the cone field $\left(\Gamma_{\sigma}, \sigma \in S\right)$.

There is a similar notion in the noncompact model. As we will have to work in this setting, let us give a more explicit description of a $\Gamma$-radial curve. First, let again $o=(0,0) \in{ }^{\gamma} S^{\prime}$. A curve $\gamma(t)=(u(t), v(t))$ is $\Gamma$-radial at $o$ if there exists $\omega \in \Omega$ and $v \in \mathbb{V}$ such that, as $t \downarrow 0$,

$$
\gamma(t)=(i t \omega, t v)+O\left(t^{2}\right)
$$

For $a \in U$ and $b \in \mathbb{V}$, the transformation

$$
(u, v) \longmapsto(u+a+2 i \Psi(v, b)+i \Psi(b, b), v+b)
$$

belongs to ${ }^{\gamma} G$ and maps the origin $o=(0,0)$ to $(a+i \Psi(b, b), b)$, which is the general point of $\gamma S^{\prime}$. So, a $\Gamma$-radial curve at the point $(a+i \Psi(b, b), b)$ is obtained from a $\Gamma$-radial curve at $(0,0)$ by this transform, hence has the following behavior as $t \downarrow 0$

$$
\gamma(t)=(a+i \Psi(b, b)+i t \omega+2 i t \Psi(v, b), b+t v)+O\left(t^{2}\right),
$$

with $\omega \in \Omega$ and $v \in \mathbb{V}$. 


\section{A geometric lemma for a Jordan algebra}

This section, except for notation, can be read independently of the first sections. We make free use of definitions, notation and results of [14]. We assume for commodity that $U$ is a simple Euclidean Jordan algebra. Denote by $e$ its unit, by $\langle.,$.$\rangle the standard invariant inner product. Let r$ be the rank of $U$. For $c$, a nonzero idempotent of $U$, we denote by $U_{1}=U_{1}(c), U_{\frac{1}{2}}=$ $U_{\frac{1}{2}}(c), U_{0}=U_{0}(c)$ the eigenspaces of $L(c)$, and let

$$
U=U_{1} \oplus U_{\frac{1}{2}} \oplus U_{0}
$$

be the corresponding Peirce decomposition of $U$. Further denote by $\Omega_{1}$ (resp. $\Omega_{0}$ ) the open cone associated to the Euclidean Jordan algebra $U_{1}$ (resp. $\left.U_{0}\right)$.

We need a couple of results, which are certainly known to experts (see exercice in $[14$, p. 59]), but we could not find accessible references.

Lemma 4.1. $\overline{\Omega_{1}}=\bar{\Omega} \cap U_{1}$.

Proof. Let $x_{1}$ be in $U_{1}$. Let $x_{1}=\lambda_{1} c_{1}+\cdots+\lambda_{s} c_{s}$ be its spectral decomposition in $U_{1}$, where $s$ is the rank of $U_{1}$ and $\left\{c_{1}, c_{2}, \ldots, c_{s}\right\}$ is a Jordan frame in $U_{1}$. So $x_{1}$ belongs to $\overline{\Omega_{1}}$ if and only if all $\lambda_{i}$ are nonnegative. But the system $\left(c_{i}\right)_{1 \leq i \leq s}$ can be completed in a Jordan frame $\left(c_{i}\right)_{1 \leq i \leq r}$ of $U$, so that the spectral decomposition of $x_{1}$ as an element of $U$ is $x_{1}=$ $\lambda_{1} c_{1}+\cdots+\lambda_{s} c_{s}+0 c_{s+1}+\cdots+0 c_{r}$. So $x_{1}$ belongs to $\bar{\Omega}$ under the same conditions on the $\left(\lambda_{i}\right)$ as before. The lemma follows.

Lemma 4.2. Let $y \in U_{\frac{1}{2}}$ and $z \in U_{0}$. Assume that $y+z \in \bar{\Omega}$. Then $y=0$ and $z \in \overline{\Omega_{0}}$.

Proof. If $U$ is of rank 1 (i.e., $U \simeq \mathbb{R}$ ), there is nothing to prove. Next assume that $U$ is of rank 2. So, let $V$ be a (finite dimensional) Euclidean vector space, and let $U=\mathbb{R} \oplus V$, with Jordan product

$$
(\lambda, v)(\mu, w)=(\lambda \mu+v \cdot w, \lambda w+\mu v),
$$

with unit element $e=(1,0)$ and inner product given by

$$
\langle(\lambda, v),(\mu, w)\rangle=\lambda \mu+v \cdot w
$$


The closed cone $\bar{\Omega}$ is characterized by

$$
(\lambda, v) \in \bar{\Omega} \Longleftrightarrow \lambda \geq 0, \lambda^{2}-v \cdot v \geq 0 .
$$

The only idempotents of interest are those of rank 1 , they are of the form $c=\left(\frac{1}{2}, v\right)$, with $v \in V$ and $\|v\|=\frac{1}{2}$. Then

$$
U_{1}=\mathbb{R}\left(\frac{1}{2}, v\right), \quad U_{\frac{1}{2}}=\{(0, w) \mid w \in V, v \cdot w=0\}, \quad U_{0}=\mathbb{R}\left(\frac{1}{2},-v\right) .
$$

Let $y=(0, w) \in U_{\frac{1}{2}}$ and $z=\left(\frac{\lambda}{2},-\lambda v\right) \in U_{0}$. Assume that $y+z=\left(\frac{\lambda}{2},-\lambda v\right.$ $+w)$ belongs to $\bar{\Omega}$. Then $\frac{\lambda^{2}}{4}-\left(\frac{\lambda^{2}}{4}+w \cdot w\right)=-w \cdot w \geq 0$. This forces $w=0$, and the assertion of the lemma follows.

Now consider the general case. Let $y \in U_{\frac{1}{2}}, z \in U_{0}$ and assume that $y+$ $z \in \bar{\Omega}$. Choose a primitive idempotent $c_{1}$ in $U_{1}$, and a primitive idempotent $c_{0}$ in $U_{0}$. As $c_{1}$ and $c_{0}$ are orthogonal, $c_{1}+c_{0}$ is an idempotent (of rank 2 ), and $P\left(c_{1}+c_{0}\right)$ is the projection on a (rank 2) Euclidean Jordan algebra, which we denote by $J$. As $\left(c_{1}, c_{0}\right)$ is a Jordan frame of $J$, the corresponding Peirce decomposition of $J$ is

$$
J=\mathbb{R} c_{1} \oplus J_{\frac{1}{2}} \oplus \mathbb{R} c_{0},
$$

where $P\left(c_{1}+c_{0}\right) U_{\frac{1}{2}}=J_{\frac{1}{2}}$. Let us make the following observation. If $x$ is any element in $\Omega$, then $P(x)$ belongs to the structure group of $U$ and preserves the cone $\Omega$. By continuity, for any $x \in \bar{\Omega}, P(x)$ maps $\bar{\Omega}$ into $\bar{\Omega}$. So this can be applied to any idempotent, as idempotents belong to $\bar{\Omega}$. So, $P\left(c_{1}+c_{0}\right)(y+z)=y_{\frac{1}{2}}+z_{0}$ belongs to $\bar{\Omega} \cap J$, which by the previous lemma is nothing but the closure in $J$ of the positive cone of $J$. Using the rank 2 result, it follows that $y_{\frac{1}{2}}=0$. But the choice of the primitive idempotents $c_{1}$ in $U_{1}$ and $c_{0}$ in $U_{0}$ is free. So, let $c=c_{1}+\cdots+c_{s}$ (resp. $e-c=c_{s+1}+\cdots+c_{r}$ ) be a Peirce decomposition of $c$ (resp. $e-c$ ) as a sum of mutually orthogonal primitive idempotents. Now

$$
U_{\frac{1}{2}}=\bigoplus_{1 \leq i \leq s<j \leq r} U_{\frac{1}{2}}\left(c_{i}+c_{j}\right)
$$

For any couple $(i, j)$ with $1 \leq i \leq s, s+1 \leq j \leq r$, the previous argument shows that $P\left(c_{i}+d_{j}\right) y=0$, and hence $y=0$. Lemma 4.2 follows.

Lemma 4.3. Let $x_{1}, x_{2} \in \bar{\Omega}$. Assume that $\left\langle x_{1}, x_{2}\right\rangle=0$. Then $x_{1} x_{2}=0$. If moreover $x_{1} \in \Omega$, then $x_{2}=0$. 
The second assertion is merely a consequence of the fact that $\Omega$ is self-adjoint. In general, there exists a Jordan frame $\left(c_{i}\right), 1 \leq i \leq r$ such that $x_{1}=\lambda_{1} c_{1}+\cdots+\lambda_{s} c_{s}$, with $\lambda_{i}>0$ for $1 \leq i \leq s$ for some $s, 1 \leq s<r$ ) (the cases where $s=0$ or $s=r$ are trivial). Let $c=c_{1}+\cdots+c_{s}$ and let $U=U_{1} \oplus U_{\frac{1}{2}} \oplus U_{0}$ be the corresponding Peirce decomposition of $U$. Let $x_{2}=\xi_{1}+\xi_{\frac{1}{2}}{ }^{2}+\xi_{0}$ be the corresponding decomposition of $x_{2}$. Now $\left\langle x_{1}, x_{2}\right\rangle=$ $\left\langle x_{1}, \xi_{1}\right\rangle=0$. But $x_{1}$ belong to the interior of the positive cone $\Omega_{1}$ of $U_{1}$, whereas $\xi_{1} \in \overline{\Omega_{1}}$. This forces $\xi_{1}=0$. So $x_{2}=\xi_{\frac{1}{2}}+\xi_{0}$, and the condition $x_{2} \in \bar{\Omega}$ implies, by Lemma 4.2 , that $\xi_{\frac{1}{2}}=0$, so that $x_{2} \in U_{0}$. The lemma follows.

Lemma 4.4. Let $u_{1} \in U_{1}$ and $u_{\frac{1}{2}} \in U_{\frac{1}{2}}$. Assume that $u_{1} u_{\frac{1}{2}}=0$ and assume that $u_{1}$ is invertible as an element of $U_{1}^{2}$. Then $u_{\frac{1}{2}}=0$.

In fact, for any element $u \in U_{1}, L(u)$ maps $U_{\frac{1}{2}}$ into itself. Moreover, let $\Phi(u)$ be the endomorphism of $U_{\frac{1}{2}}$ defined by

$$
U_{\frac{1}{2}} \ni v \longmapsto \Phi(u) v=2 u v \in U_{\frac{1}{2}} .
$$

Then $\Phi$ is known to be a representation of $U_{1}$ on $U_{\frac{1}{2}}$ (see [14]). In particular,

$$
\Phi\left(u u^{\prime}\right)=\frac{1}{2}\left(\Phi(u) \Phi\left(u^{\prime}\right)+\Phi\left(u^{\prime}\right) \Phi(u)\right), \quad \Phi(c)=\mathrm{Id} .
$$

Let $u \in U_{1}$ be invertible in $U_{1}$. Its inverse in $U_{1}$ is of the form $u^{\prime}=p(u)$, where $p \in \mathbb{R}[X]$. As $\Phi$ is a representation, one has $\Phi(p(u))=p(\Phi(u))$, and hence $\Phi(u)$ is invertible and $\Phi(u)^{-1}=\Phi\left(u^{\prime}\right)$. The lemma follows.

Lemma 4.5. Let $b \in U$ and $y \in \bar{\Omega}$. Assume that $\langle b y, b\rangle=0$. Then $b y=0$.

Proof. Let $b \in U$ and $y \in \bar{\Omega}$, and let $b=\sum_{j=1}^{k} \lambda_{j} c_{j}$ be the spectral expression of $b$, where the $\left(c_{j}\right)_{1 \leq j \leq k}$ are orthogonal (non-necessarily primitive) idempotents, and the $\left(\lambda_{j}\right)_{1 \leq j \leq k}$ are the nonzero distinct eigenvalues of $b$. Then $b^{2}=\sum_{j=1}^{k} \lambda_{j}^{2} c_{j}$, so that the condition $\langle b y, b\rangle=0$ implies

$$
\sum_{j=1}^{k} \lambda_{j}^{2}\left\langle y, c_{j}\right\rangle=0
$$

As for any $j, 1 \leq j \leq k, \lambda_{j} \neq 0$ and $\left\langle y, c_{j}\right\rangle \geq 0$, (4.1) forces $\left\langle y, c_{j}\right\rangle=0$ for every $j, 1 \leq j \leq k$. As $y, c_{j} \in \bar{\Omega}$, this in turn implies, by Lemma $4.3, c_{j} y=0$ for any $j$ and so $b y=0$. 
For $c$ any idempotent of $U$, use the notation $\operatorname{det}_{1}$ (resp. $\operatorname{det}_{0}$ ) for the determinant of the Jordan algebra $U_{1}$ (resp. $U_{0}$ ). Let us complexify the Peirce decomposition of $U$ to obtain

$$
\mathbb{U}=\mathbb{U}_{1}(c) \oplus \mathbb{U}_{\frac{1}{2}}(c) \oplus \mathbb{U}_{0}(c) .
$$

The tube associated to $U$ is the open set $T_{\Omega}$ in $\mathbb{U}$ defined by

$$
T_{\Omega}=U+i \Omega=\{z=x+i y \mid x, y \in U, y \in \Omega\} .
$$

Observe that any element of $T_{\Omega}$ is invertible in $\mathbb{U}$. This property is no longer true for all points in the boundary of $T_{\Omega}$, but some substitute is valid. The following technical proposition will be the key point to justify the limit process (1.5) needed for handling singular triples.

Proposition 4.6. Let $z \in \overline{T_{\Omega}}, z \neq 0$. There exists an idempotent $c \in U$ such that $z \in \mathbb{U}_{1}(c)$ and $z$ is invertible as an element of $\mathbb{U}_{1}(c)$.

Proof. If $z$ is invertible in $\mathbb{U}$, then take $c=e$ and the corresponding properties are satisfied. So we may (and hence do) assume that $L(z)$ is not invertible. Hence, there exists $u \in \mathbb{U}, u \neq 0$ such that $z u=0$. Let $z=x+i y$ and $u=a+i b$, with $x, y, a, b \in U$. Then $z u=0$ reads

$$
x a-y b=0, \quad x b+y a=0 .
$$

As $y \in \bar{\Omega}$,

$$
\langle x a, b\rangle=\langle y b, b\rangle \geq 0, \quad\langle x b, a\rangle=-\langle y a, a\rangle \leq 0 .
$$

But $\langle x a, b\rangle=\langle x b, a\rangle$, so that $0=\langle y b, b\rangle$. From Lemma 4.5 follows $y b=0$. Similarly, $y a=0$, so that $x a=y b=0$ and $x b=y a=0$. Hence $z a=z b=0$. But $(a, b) \neq(0,0)$, and so without loosing any generality, we may assume that $u \in U$.

So assume $u \in U, u \neq 0$ such that $x u=y u=0$. Using the spectral decomposition of $y$, one can find an idempotent $d \in U$ such that $y \in U_{1}(d)$ and $y$ is invertible in $U_{1}(d)$. Let $u=u_{1}+u_{\frac{1}{2}}+u_{0}$ be the Peirce decomposition of $u$ with respect to $c$. Then

$$
0=y u=y\left(u_{1}+u_{\frac{1}{2}}+u_{0}\right)=y u_{1}+y u_{\frac{1}{2}} .
$$

But $y u_{1} \in U_{1}(d)$ and $y u_{\frac{1}{2}} \in U_{\frac{1}{2}}(d)$. This forces

$$
y u_{1}=0, \quad y u_{\frac{1}{2}}=0 .
$$


The second equality, thanks to Lemma 4.4, implies $u_{\frac{1}{2}}=0$. Moreover, as $y$ belongs to $U_{1} \cap \bar{\Omega}$ and is invertible in $U_{1}(d), y$ is in the open cone $\Omega_{1}=\Omega_{1}(d)$ of $U_{1}(d)$, so that the first equality implies that $u_{1}=0$. So $u$ belongs to $U_{0}(d)$. As $U_{0}(d)$ is a Euclidean Jordan algebra, there exists an idempotent $f \in U_{0}(d) \subset U$ such that $u \in U_{1}(f)$ and $u$ is invertible in $U_{1}(f)$. Now let $c=e-f$. As $f \in U_{0}(d)$ and $y \in U_{1}(d), f y=0$ and hence $y \in U_{1}(c)$, whereas $u \in U_{0}(c)$. Now let $x=x_{1}+x_{\frac{1}{2}}+x_{0}$ be the Peirce decomposition of $x$ with respect to the idempotent $c$. As before, the equality $x u=0$ implies $x_{\frac{1}{2}} u=0$, and the fact that $u$ is invertible in $U_{0}(c)=U_{1}(f)$ implies $x_{\frac{1}{2}}=0$. ${ }^{\frac{1}{2}}$ Hence we get

$$
z=x_{1}+i y+x_{0}
$$

where $x_{1}, y \in U_{1}(c)$ and $x_{0} \in U_{0}(c)$.

Among all idempotents $c$ in $U$ such that $z$ can be written as $z=x_{1}+$ $i y+x_{0}$ with $x_{1}, y \in U_{1}(c)$ and $x_{0} \in U_{0}(c)$, choose $c$ minimal. In the corresponding decomposition $z=x_{1}+i y+x_{0}$, the element $x_{1}+i y$ has to be invertible in $\mathbb{U}_{1}(c)$. Otherwise, we could repeat for the Euclidean algebra $U_{1}(c)$ and the element $z_{1}=x_{1}+i y$ the first part of the proof, to get an idempotent $c^{\prime} \neq c$ in $U_{1}(c)$ such that $z_{1}=x_{1}^{\prime}+i y+x_{0}^{\prime}$, with $x_{1}^{\prime}, y \in U_{1}\left(c^{\prime}\right)$ and $x_{0}^{\prime} \in U_{0}\left(c^{\prime}\right)$. But then, one can write $z=x_{1}^{\prime}+i y+x_{0}^{\prime}+x_{0}$, where $x_{1}^{\prime}, y \in U_{1}\left(c^{\prime}\right)$ and $x_{0}^{\prime}+x_{0} \in U_{0}\left(c^{\prime}\right)$, hence contradicting the minimality of $c$.

So there exists an idempotent $c \in U$ such that $z=x_{1}+i y+x_{0}$, where $x_{1}+i y$ is invertible in $\mathbb{U}_{1}(c)$, and $x_{0} \in U_{0}(c)$. As $0=\operatorname{det}(z)=\operatorname{det}_{1}\left(x_{1}+\right.$ $i y) \operatorname{det}_{0} x_{0}$, necessarily $\operatorname{det}_{0} x_{0}=0$. If $x_{0}=0$, then there is nothing more to prove. If not, there exists an idempotent $c^{\prime} \in U_{0}(c)$, such that $c^{\prime} x_{0}=$ $x_{0}$ and $x_{0}$ is invertible in $U_{1}\left(c^{\prime}\right)$. Now $c^{\prime \prime}=c+c^{\prime}$ is an idempotent in $U$, $c^{\prime \prime} z=z$, and as $x_{1}+i y$ is invertible in $\mathbb{U}_{1}(c)$ and $x_{0}$ is invertible in $U_{1}\left(c^{\prime}\right)$, hence $z=\left(x_{1}+i y\right)+x_{0}$ is invertible in $\mathbb{U}_{1}\left(c^{\prime \prime}\right)$. This finishes the proof of Proposition 4.6.

Remark 4.7. Let $U=\operatorname{Sym}(2, \mathbb{R})$ be the Euclidean Jordan algebra of $2 \times 2$ symmetric matrices with real entries. Consider the element

$$
z=\left(\begin{array}{cc}
1 & i \\
i & -1
\end{array}\right)=\left(\begin{array}{cc}
1 & 0 \\
0 & -1
\end{array}\right)+i\left(\begin{array}{ll}
0 & 1 \\
1 & 0
\end{array}\right) .
$$

As $z \neq 0$ and $z^{2}=0$, there is no idempotent $c \in U$ such that $z$ is invertible in $\mathbb{U}_{1}(c)$. This shows that some condition (like $z \in \overline{T_{\Omega}}$ ) is needed in order that the conclusion of Proposition 4.6 be valid. 
To illustrate Proposition 4.6, let us consider the case where $U$ is the Euclidean Jordan algebra of rank 2 and dimension $1+d$ associated to the Lorentz cone in $\mathbb{R}^{1+d}$. So $U=\mathbb{R} \oplus V$, where $V$ is a Euclidean vector space of dimension $d$, with inner product denoted by $\langle.,$.$\rangle . The Jordan$ product is

$$
(\lambda, v)(\mu, w)=(\lambda \mu+\langle v, w\rangle, \lambda w+\mu v)
$$

with inner product given by

$$
\langle(\lambda, v),(\mu, w)\rangle=\lambda \mu+\langle v, w\rangle
$$

and determinant function given by

$$
\operatorname{det}(\lambda, v)=\lambda^{2}-\langle v, v\rangle
$$

The cone $\Omega$ is

$$
\Omega=\left\{(\lambda, v) \mid \lambda^{2}-\langle v, v\rangle>0, \lambda>0\right\}
$$

Let $z \in \overline{T_{\Omega}}, z \neq 0$, and assume $\operatorname{det} z=0$. Then the content of Proposition 4.2 in this case is that $z$ is a complex multiple of an idempotent of rank 1, that is to say of the form

$$
z=\zeta\left(\frac{1}{2}, \frac{1}{2} f\right), \text { where } f \in U,\langle f, f\rangle=1 \quad \text { and } \quad \zeta \in \mathbb{C}, \zeta \neq 0, \Im \zeta \geq 0 \text {. }
$$

This can be shown more directly as follows. Let $z=x+i y=(\lambda+i \mu, v+$ $i w)$. The condition $\operatorname{det} z=0$ is equivalent to

$$
\lambda^{2}-\langle v, v\rangle=\mu^{2}-\langle w, w\rangle, \quad \lambda \mu=\langle v, w\rangle .
$$

As $y \in \bar{\Omega},\langle w, w\rangle^{\frac{1}{2}} \leq|\mu|$, so that also $\langle v, v\rangle^{\frac{1}{2}} \leq|\lambda|$. But, from CauchySchwarz inequality,

$$
|\lambda \mu|=|\langle v, w\rangle| \leq\langle v, v\rangle^{\frac{1}{2}}\langle w, w\rangle^{\frac{1}{2}} \leq|\lambda||\mu|,
$$

so that the equality is achieved, and hence $v$ and $w$ are proportional. As $\mu=\langle w, w\rangle^{\frac{1}{2}}$ and $|\lambda|=\langle v, v\rangle^{\frac{1}{2}}$, the statement easily follows. 
Proposition 4.8. Let $c$ be an idempotent in $U$ and $u$ be an invertible element of $\mathbb{U}_{1}(c)$. Let $\gamma:[0,1] \rightarrow \mathbb{U}$ be a smooth curve such that

$$
\gamma(0)=u, \quad \dot{\gamma}(0)=z_{1}+z_{\frac{1}{2}}+\omega_{0}
$$

where $z_{1} \in \mathbb{U}_{1}(c), z_{\frac{1}{2}} \in \mathbb{U}_{\frac{1}{2}}(c)$ and $\omega_{0} \in \Omega_{0}$. Then $t \longmapsto \arg \operatorname{det}(\gamma(t))$ is welldefined for sufficiently small positive $t$. Furthermore, the limit $t \rightarrow 0$ exists and is independent of $z_{1}, z_{\frac{1}{2}}$ and $\omega_{0}$.

Proof. From the assumptions, for $t>0$, one has

$$
\gamma(t)=u+t z_{1}+t z_{\frac{1}{2}}+t \omega_{0}+O\left(t^{2}\right)
$$

For $z \in \mathbb{U}_{\frac{1}{2}}(c)$, we let $\tau(z)=\exp (2 z \square c)$ be the Frobenius transformation (see $[14$, ch. VI]). The main property we will use is that for any $v \in \mathbb{U}$,

$$
\operatorname{det}(\tau(z) v)=\operatorname{det} v
$$

Recall the following lemma (see [3] Lemma 2.2).

Lemma 4.9. Let $\xi_{1}$ be an invertible element in $\mathbb{U}_{1}(c)$. Then, the map

$$
\begin{gathered}
\left(\eta_{1}, z, \eta_{0}\right) \longmapsto \zeta=\left(\zeta_{1}, \zeta_{\frac{1}{2}}, \zeta_{0}\right)=\tau(z)\left(\eta_{1}+\eta_{0}\right), \\
\mathbb{U}_{1}(c) \times \mathbb{U}_{\frac{1}{2}}(c) \times \mathbb{U}_{0}(c) \longrightarrow \mathbb{U}
\end{gathered}
$$

is a local diffeomorphism near $\left(\xi_{1}, 0,0\right)$. Its inverse is given by

$$
\begin{gathered}
\eta_{1}(\zeta)=\zeta_{1} \\
z(\zeta)=\Phi\left(\zeta_{1}\right)^{-1} \zeta_{\frac{1}{2}} \\
\eta_{0}(\zeta)=\zeta_{0}-2 L(e-c) L(z(\zeta))^{2} \zeta_{1} .
\end{gathered}
$$

As $u$ is invertible in $\mathbb{U}_{1}(c)$, we may apply the lemma to $\xi_{1}=u$. Hence, for $t$ small enough,

$$
\gamma(t)=\tau(z(\gamma(t)))\left(\eta_{1}(\gamma(t))+\eta_{0}(\gamma(t))\right)
$$

so that, using (4.5)

$$
\operatorname{det}(\gamma(t))=\operatorname{det}_{1}\left(\eta_{1}(\gamma(t))\right) \operatorname{det}_{0}\left(\eta_{0}(\gamma(t))\right) .
$$

As $t \longrightarrow 0$,

$$
\eta_{1}(\gamma(t)) \longrightarrow u
$$


and hence

$$
\operatorname{det}_{1}\left(\eta_{1}(\gamma(t))\right) \longrightarrow \operatorname{det}_{1}(u) \neq 0,
$$

so that any determination of the corresponding argument has a limit when $t \longrightarrow 0$, and the limit does not depend on $\dot{\gamma}(0)$.

Observe further that $z(\gamma(t))=O(t)$ and hence $\eta_{0}(\gamma(t))=t \omega_{0}+O\left(t^{2}\right)$ as $t \downarrow 0$, so that $\arg \operatorname{det}_{0}\left(\eta_{0}(\gamma(t))\right)$ tends to $0(\bmod 2 \pi)$ as $t \downarrow 0$. This finishes the proof of the proposition.

\section{Symplectic area of geodesic triangles and the invariant for triples}

Recall the setting of Sections 2 and 3. On $\mathcal{D}$, there exists a Hermitian metric which is invariant under $G$. It can be defined using the Bergman kernel of the domain, but there is a normalization of the metric which is more convenient for geometric purposes, by requiring that the minimal value of the holomorphic sectional curvature to be -1 (see [4] for details). The corresponding Kähler form $\omega$ is invariant under $G$ and closed.

Given two points $z_{1}, z_{2} \in \mathcal{D}$, there is a unique geodesic segment connecting $z_{1}$ to $z_{2}$. So, for any triple of points $z_{1}, z_{2}, z_{3}$ in $\mathcal{D}$, we may form the oriented geodesic triangle $T\left(z_{1}, z_{2}, z_{3}\right)$. Let $\Sigma$ be any surface in $\mathcal{D}$ with (oriented) boundary equal to $T\left(z_{1}, z_{2}, z_{3}\right)$. Then integrate the 2 -form $\omega$ on $\Sigma$ to get the symplectic area of the triangle

$$
\varphi\left(z_{1}, z_{2}, z_{3}\right)=\int_{\Sigma} \omega
$$

As the Kähler form $\omega$ is closed, this expression does not depend on the surface $\Sigma$, but merely on its boundary, and hence defines a function $\varphi\left(z_{1}, z_{2}\right.$, $z_{3}$ ). It turns out that the symplectic area can be computed explicitly (see $[4$, $17])$. Let $k(z, w): \mathcal{D} \times \mathcal{D} \longrightarrow \mathbb{C}$ be the normalized automorphy kernel of $\mathcal{D}$ (see [4] for definition). Then, for any triple $\left(z_{1}, z_{2}, z_{3}\right) \in \mathcal{D}$,

$$
\varphi\left(z_{1}, z_{2}, z_{3}\right)=-\left(\arg k\left(z_{1}, z_{2}\right)+\arg k\left(z_{2}, z_{3}\right)+\arg k\left(z_{3}, z_{1}\right)\right),
$$

where $\arg k(z, w)$ is the unique determination of the argument of $k(z, w)$ which is continuous on $\mathcal{D} \times \mathcal{D}$ and which is 0 on the diagonal of $\mathcal{D} \times \mathcal{D}$ (recall that $k(z, z)>0$ for any $z \in \mathcal{D}$ ).

Theorem 5.1. Let $\left(\sigma_{1}, \sigma_{2}, \sigma_{3}\right) \in S^{3}$. Then $\varphi\left(z_{1}, z_{2}, z_{3}\right)$ has a limit when, for any $j, 1 \leq j \leq 3, z_{j} \rightarrow \sigma_{j}$ along a $\Gamma$-radial curve. 
Proof. By using the action of $G$ and the invariance of the symplectic area under $G$, we may choose a maximal tripotent $e \in \mathbb{W}$ and assume that the Cayley transform $\gamma=\gamma_{e}$ is defined at $\sigma_{1}, \sigma_{2}$ and $\sigma_{3}$. So, it is equivalent to prove the analogous statement in the noncompact realization of $\mathcal{D}$. The normalized automorphy kernel $\gamma k$ in the unbounded picture reads: for $(z, v)$ and $\left(z^{\prime}, v^{\prime}\right)$ in ${ }^{\gamma} \mathcal{D}$

$$
{ }^{\gamma} k\left((u, v),\left(u^{\prime}, v^{\prime}\right)\right)=\left(\operatorname{det}\left(\frac{u-\overline{u^{\prime}}}{2 i}-\Psi\left(v, v^{\prime}\right)\right)\right)^{-2} .
$$

The formula (5.2) comes from a general formula for the Bergman kernel of a Siegel domain of the second kind (see [11 ch. III, section 6, ex 3]), after adjusting the result to the normalization chosen for the corresponding metric.

As the expression for ${ }^{\gamma} \varphi$ is a sum of three similar terms, it suffices to prove that each such term has a limit, and we may even assume that the first point (say) is the base point $o$ in ${ }^{\gamma} S^{\prime}$. So, Theorem 5.1 is a consequence of the following lemma.

Lemma 5.2. Let $\sigma=(a+i \Psi(b, b), b) \in{ }^{\gamma} S^{\prime}$. Let $\lambda \in \Omega, w \in \mathbb{V}$ and let

$$
\lambda(t)=(u(t), v(t))=(i t \lambda, t w)+O\left(t^{2}\right)
$$

be $a$-radial curve at the base point $o$. Let $\mu \in \Omega, v \in \mathbb{V}$ and let

$$
\mu(t)=\left(u^{\prime}(t), v^{\prime}(t)\right)=(a+i \Psi(b, b)+i t \mu+2 i t \Psi(v, b), b+t v)+O\left(t^{2}\right)
$$

be a $\Gamma$-radial curve at $\sigma\left(c f\right.$. (3.8)). Then $\arg { }^{\gamma} k(\lambda(t), \mu(t))$ has a limit as $t \downarrow 0$. The limit is independent of $\lambda, w, \mu$ and $v$.

Proof. Define

$$
\gamma(t)=\frac{u(t)-\overline{u^{\prime}(t)}}{2 i}-\Psi\left(v(t), v^{\prime}(t)\right)
$$

By an easy computation,

$$
\gamma(t)=\frac{-a+i \Psi(b, b)}{2 i}+t\left(\frac{\lambda+\mu}{2}+\Psi(v, b)-\Psi(w, b)\right)+O\left(t^{2}\right) .
$$

Observe that $z=-a+i \Psi(b, b) \in \overline{T_{\Omega}}$. Hence, from Proposition 4.6* , there exists an idempotent $c \in U$, such that $z \in \mathbb{U}_{1}(c)$ and $z$ is invertible in $\mathbb{U}_{1}(c)$.

${ }^{*}$ If $z=0$, use $c=0$ for the proof of the lemma. 
Use (2.11) to obtain

$$
\Psi(\Phi(e-c) b, \Phi(e-c) b)=P(e-c) \Psi(b, b)=0 .
$$

But, thanks to (2.10), this implies $\Phi(e-c) b=0$. In turn, using (2.11) again, this implies

$$
P(e-c) \Psi(v, b)=\Psi(\Phi(e-c) v, \Phi(e-c) b)=0,
$$

and so

$$
\Psi(v, b) \in \mathbb{U}_{1}(c) \oplus \mathbb{U}_{\frac{1}{2}}(c),
$$

and similarly for $\Psi(w, b)$. Let $\lambda=\lambda_{1}+\lambda_{\frac{1}{2}}+\lambda_{0}$ (resp. $\left.\mu=\mu_{1}+\mu_{\frac{1}{2}}+\mu_{0}\right)$ be the Peirce decomposition of $\lambda$ (resp. $\mu$ ) with respect to $c$. Then (5.3) can be rewritten as

$$
\gamma(t)=\frac{-a+i \Psi(b, b)}{2 i}+t z_{1}+t z_{\frac{1}{2}}+t\left(\frac{\lambda_{0}+\mu_{0}}{2}\right)+O\left(t^{2}\right)
$$

where $z_{1} \in \mathbb{U}_{1}(c), z_{\frac{1}{2}} \in \mathbb{U}_{\frac{1}{2}}(c)$. As $\lambda_{0}+\mu_{0} \in \Omega_{0}$, the conditions of Proposition 4.8 are fulfilled, and so we may conclude that $\arg \operatorname{det} \gamma(t)$ has a limit as $t \downarrow 0$, not depending on $\lambda, \mu$ nor on $v, w$, and hence the same is true for $\arg \gamma k(\lambda(t), \mu(t))$.

Following Theorem 5.1, define $\iota: S \times S \times S \longrightarrow \mathbb{R}$ by

$$
\iota\left(\sigma_{1}, \sigma_{2}, \sigma_{3}\right)=\frac{1}{\pi} \lim _{z_{j} \longrightarrow \sigma_{j}} \varphi\left(z_{1}, z_{2}, z_{3}\right),
$$

where, for each $j, 1 \leq j \leq 3, z_{j}$ tends to $\sigma_{j}$ along a $\Gamma$-radial curve.

Theorem 5.3. The function $\iota\left(\sigma_{1}, \sigma_{2}, \sigma_{3}\right)$ satisfies the following relations:

(i) $\iota\left(g \sigma_{1}, g \sigma_{2}, g \sigma_{3}\right)=\iota\left(\sigma_{1}, \sigma_{2}, \sigma_{3}\right)$

(ii) $\iota\left(\sigma_{\pi(1)}, \sigma_{\pi(2)}, \sigma_{\pi(3)}\right)=\operatorname{sgn}(\pi) \iota\left(\sigma_{1}, \sigma_{2}, \sigma_{3}\right)$

(iii) $\iota\left(\sigma_{1}, \sigma_{2}, \sigma_{3}\right)=\iota\left(\sigma_{1}, \sigma_{2}, \sigma_{4}\right)+\iota\left(\sigma_{2}, \sigma_{3}, \sigma_{4}\right)+\iota\left(\sigma_{3}, \sigma_{1}, \sigma_{4}\right)$

(iv) $-r \leq \iota\left(\sigma_{1}, \sigma_{2}, \sigma_{3}\right) \leq r$

for all $\sigma_{1}, \sigma_{2}, \sigma_{3}, \sigma_{4} \in S, g \in G$ and $\pi$ any permutation of $\{1,2,3\}$.

Proof. The function $\varphi$ satisfies the same properties on $\mathcal{D} \times \mathcal{D} \times \mathcal{D}$, as was shown in [4]. The corresponding properties for $\iota$ are clearly preserved through the limit process. 


\section{Extremal values of Cartan's invariant}

The triples corresponding to the extremal values of the invariant $\iota$ have a geometric description. For the unit sphere in $\mathbb{C}^{2}$, this was observed by Cartan. For general bounded symmetric domains, a characterization of the extremal triples was obtained in [4], but only for mutually transverse triples (see also [18]). Here we treat the general case.

Definition 6.1. ${ }^{2}$ Let $\Delta=\{z \in \mathbb{C},|z|<1\}, \mathcal{D}$ a bounded symmetric domain and $\rho: \Delta \longrightarrow \mathcal{D}$ a holomorphic equivariant totally geodesic map. Then $\rho$ is said to be tight if $\rho$ maps the boundary $\Sigma=\{\sigma \in \mathbb{C},|\sigma|=1\}$ into the Shilov boundary $S$ of $\mathcal{D}$.

If $e \in S$, then the map

$$
\rho: \Delta \longrightarrow \mathcal{D}, \quad z \longmapsto z e
$$

is a tight holomorphic embedding, and up to composition by an element of $G$, every tight holomorphic imbedding can be realized in this way (cf. [11, ch. III]).

Theorem 6.2. Let $\sigma_{1}, \sigma_{2}, \sigma_{3} \in S$ and assume that $\iota\left(\sigma_{1}, \sigma_{2}, \sigma_{3}\right)=r$. Then, for $1 \leq i \neq j \leq 3, \sigma_{i} \top \sigma_{j}$, and there exists a unique tight holomorphic totally geodesic imbedding $\rho: \Delta \longrightarrow \mathcal{D}$ such that

$$
\sigma_{1}=\rho(+1), \quad \sigma_{2}=\rho(-1), \quad \sigma_{3}=\rho(-i) .
$$

Only the first statement (the fact that a triple which realizes the maximal value of the invariant $\iota$ has to be mutually transverse) is new. The second statement, assuming the transversality property, was proved in [4]. For the proof of the first statement, we need to recall a few results, which we state as lemmas. Recall first that, by Bruhat's theory, the $G$-orbits in $S \times S$ are easy to analyze. There are exactly $r+1$ orbits. Here is a more precise statement (see e.g. [19] for a proof).

Lemma 6.3. Let $c_{1}, c_{2}, \cdots, c_{r}$ be a Jordan frame of $\mathbb{W}$. For $0 \leq k \leq r$, let

$$
\varepsilon_{k}=c_{1}+c_{2}+\cdots+c_{k}-c_{k+1}-\cdots-c_{r},
$$

and $e=\varepsilon_{r}=c_{1}+c_{2}+\cdots+c_{r}$. Any pair $\left(\sigma_{1}, \sigma_{2}\right) \in S \times S$ is G-conjugate to some pair $\left(e, \varepsilon_{k}\right)$ for a unique $k, 0 \leq k \leq r$.

\footnotetext{
${ }^{2}$ The definition is easily seen to coincide with the definition used in [4].
} 
Set $\mu\left(\sigma_{1}, \sigma_{2}\right)=k$ (transversality index) if the pair $\left(\sigma_{1}, \sigma_{2}\right)$ is $G$-conjugate to $\left(e, \varepsilon_{k}\right)$.

Lemma 6.4. Let $\mathcal{D}$ be a bounded symmetric domain of tube type, of rank $r$. Let $\sigma_{1}, \sigma_{2}, \sigma_{3} \in S \times S \times S$ and assume $\mu\left(\sigma_{1}, \sigma_{2}\right)=k$. Then

$$
\left|\iota\left(\sigma_{1}, \sigma_{2}, \sigma_{3}\right)\right| \leq r-k \text {. }
$$

This a consequence of a geometric result proved in [19]. In fact, up to $G$-action, there exists a Jordan frame $c_{1}, c_{2}, \ldots, c_{r}$ such that

$$
\begin{aligned}
\sigma_{1} & =c_{1}+c_{2}+\cdots+c_{r}, \quad \sigma_{2}=c_{1}+\cdots+c_{k}-c_{k+1}-\cdots-c_{r}, \\
\sigma_{3} & =\sum_{j=1}^{r} e^{i \theta_{j}} c_{j},
\end{aligned}
$$

where for $1 \leq j \leq r \theta_{j} \in \mathbb{R} / 2 \pi \mathbb{Z}$. Now,

$$
\iota\left(\sigma_{1}, \sigma_{2}, \sigma_{3}\right)=\sum_{j=k+1}^{r} \iota\left(1,-1, e^{i \theta_{j}}\right),
$$

where we use the same symbol $\iota$ for the Maslov index on the unit circle in C. Lemma 6.4 follows immediately.

Lemma 6.5. Let $\mathcal{D}$ be a bounded symmetric domain of tube type, of rank $r$. Let $\sigma_{1}, \sigma_{2} \in S \times S$ and let $\mu\left(\sigma_{1}, \sigma_{2}\right)=k$. For $z \in \mathcal{D}$, let $\varphi$ be the function on $S \times S \times \mathcal{D}$ defined by

$$
\varphi\left(\sigma_{1}, \sigma_{2}, z\right)=\lim _{z_{1} \longrightarrow \sigma_{1}, z_{2} \longrightarrow \sigma_{2}} \varphi\left(z_{1}, z_{2}, z\right),
$$

where the limit is taken in the $\Gamma$-radial sense. Then

$$
\left|\varphi\left(\sigma_{1}, \sigma_{2}, z\right)\right|<(r-k) \pi
$$

for any $z \in \mathcal{D}$.

As a function of $z, \psi(z)=\varphi\left(\sigma_{1}, \sigma_{2}, z\right)$ is pluriharmonic in $\mathcal{D}$ (see the argument in [4]). The function $\psi$ is bounded by $r \pi$ and has $\Gamma$-radial limits at all points of the Shilov boundary $S$, and, by Lemma 6.4, satisfies $|\psi(\sigma)| \leq$ $(r-k) \pi$ for all $\sigma \in S$. A maximum principle then applies (cf. [20]), which implies that the same bound is valid on $\overline{\mathcal{D}}$. The lemma follows as the maximum cannot be reached in $\mathcal{D}$. 
Lemma 6.6. Let $\mathcal{D}$ be a bounded symmetric domain. Let $\sigma_{1}, \sigma_{2} \in S \times S$ and let $\mu\left(\sigma_{1}, \sigma_{2}\right)=k$. Then, for any $\sigma_{3} \in S$,

$$
\left|\iota\left(\sigma_{1}, \sigma_{2}, \sigma_{3}\right)\right| \leq r-k
$$

Proof. From Lemma 6.3, we may assume that $\sigma_{1}=e$ and $\sigma_{2} \in \mathbb{W}_{2}(e)$. For $z \in \mathcal{D}$, let $z^{\prime}$ be the orthogonal projection of $z$ on $\mathbb{W}_{2}(e)$. Let $\mathcal{D}^{\prime}=\mathcal{D} \cap$ $\mathbb{W}_{2}(e)$. It is a bounded symmetric domain of tube type, of the same rank $r$ as $\mathcal{D}$. Moreover, $z^{\prime} \in \mathcal{D}^{\prime}$ and (cf. [4])

$$
\varphi\left(\sigma_{1}, \sigma_{2}, z\right)=\varphi^{\prime}\left(\sigma_{1}, \sigma_{2}, z^{\prime}\right)
$$

where $\varphi^{\prime}$ is the symplectic area for the tube-type domain $\mathcal{D}^{\prime}$. Now Lemma 6.5 implies $\left|\varphi^{\prime}\left(\sigma_{1}, \sigma_{2}, z^{\prime}\right)\right| \leq(r-k) \pi$, and hence $\left|\varphi\left(\sigma_{1}, \sigma_{2}, z\right)\right| \leq(r-k) \pi$. The inequality (6.1) is obtained by letting $z$ tend to $\sigma_{3}$ along a $\Gamma$-radial curve.

The proof of Theorem 6.2 is now easy. If $\iota\left(\sigma_{1}, \sigma_{2}, \sigma_{3}\right)=r$, then $\sigma_{1} \top \sigma_{2}$, because of Lemma 6.6, and similarly $\sigma_{2} \top \sigma_{3}$ and $\sigma_{3} \top \sigma_{1}$. This means that a triple which realizes the maximum of the Cartan invariant must be mutually transverse. Then Theorem 6.2 is a consequence of Theorem 4.7 in [4]. Needless to say, there is a similar result for triples in $S$ which satisfy $\iota\left(\sigma_{1}, \sigma_{2}, \sigma_{3}\right)=-r$. For applications of this result, see [8] or [18].

\section{References}

[1] E. Cartan, Sur le groupe de la géométrie hypersphérique, Comm. Math. Helv. 4 (1932), 158-171.

[2] J.-L. Clerc and B. Ørsted, The Maslov index revisited, Transform. Groups 6 (2001), 303-320.

[3] J.-L. Clerc, L'indice de Maslov généralisé, J. Math. Pures Appl. 83 (2004), 99-114.

[4] J.-L. Clerc and B. Ørsted, The Gromov norm of the Kaehler class and the Maslov index, Asian J. Math. 7 (2003), 269-296.

[5] W. M. Goldman, Complex hyperbolic geometry, Oxford mathematical monographs, Clarendon Press, Oxford, 1999.

[6] G. Lion and M. Vergne, The Weil representation, Maslov index and theta series, Progress in Mathematics, Birkhäuser, Boston, 61980. 
[7] J.-L. Clerc, A triple ratio on the unitary Stiefel manifold, L'Enseignement Mathématique 48 (2002), 51-71.

[8] M. Burger and A. Iozzi, Bounded Kähler class rigidity of actions on Hermitian symmetric spaces, Ann. Sci. Norm. Sup. 37 (2004), no. 4, 77-103.

[9] O. Loos, Bounded symmetric domains and Jordan pairs, Mathematical lectures, University of California at Irvine, 1977.

[10] J. A. Wolf and A. Korányi A. Generalized Cayley transformations of bounded symmetric domains, Amer. J. Math. 98 (1965), 899-939.

[11] I. Satake, Algebraic structures of symmetric domains, Kanô Memorial Lectures 4, Iwanami Shoten and Princeton University Press, Princeton, 1980 .

[12] A. Korányi, Function spaces on bounded symmetric domains, Analysis and Geometry on complex homogeneous domains, part III, 183-281, Progress in Mathematics, Birkhäuser, Boston, 185, 2000.

[13] M. Koecher, An elementary approach to bounded symmetric domains, Lecture notes, Rice University, Houston, Texas, 1969.

[14] J. Faraut and A. Korányi, Analysis on symmetric cones, Oxford mathematical monographs, Clarendon Press, Oxford, 1994.

[15] S. Kaneyuki, On the causal structure of the Shilov boundaries of symmetric domains, Prospects in complex geometry (Katata and Kyoto, 1989), Lecture notes in math. 1468, 127-159, Springer, Berlin, 1999.

[16] J. Hilgert and G. Ólafsson, Causal symmetric spaces, geometry and analysis, Perspectives in mathematics, Academic Press, San Digo, 18, 1997.

[17] A. Domic and D. Toledo, The Gromov norm of the Kaehler class of symmetric domains, Math. Ann. 276 (1987) 425-432.

[18] A. Wienhard, Bounded cohomology and geometry, Bonner Mathematische Schriften 368, Bonn, 2004. 
[19] J.-L. Clerc and K.-H. Neeb, Orbits of triples in the Shilov boundary of a bounded symmetric domain, Transform. Groups 11 (2006), 387-426.

[20] J.-L. Clerc and B. Ørsted, Corrigendum to "The Gromov norm of the Kaehler class and the Maslov index”, Asian J. Math. 8 (2004), 391-394.

Institut Elie Cartan U.M.R. 7502

NANCY UnIVERSITÉ, CNRS, INRIA

B.P. 239

54506 VANDEUVRE-LÈS-NANCY CEDEX

FRANCE

E-mail address: clerc@iecn.u-nancy.fr

Received June 28, 2005 
